\title{
The circulation of human astrovirus genotypes in the Central West Region of Brazil
}

\author{
Paula Andreia Silva ${ }^{1,7}$, Rodrigo Alessandro Tôgo Santos ${ }^{1}$, Paulo Sérgio Sucasas Costa ${ }^{2}$, \\ José Marcus Sócrates Teixeira ${ }^{3}$, Loreny Gimenes Giugliano ${ }^{4}$, Márcia Sueli Assis Andreasi ${ }^{5}$, \\ José Paulo Gagliardi Leite ${ }^{6}$, Ecakart Schreier ${ }^{7}$, Divina das Dôres de Paula Cardoso ${ }^{1 /+}$
}

\begin{abstract}
${ }^{1}$ Laboratório de Virologia, Instituto de Patologia Tropical e Saúde Pública ${ }^{2}$ Departamento de Pediatria, Faculdade de Medicina, Universidade Federal de Goiás, Rua 235 com 1aa Avenida, Setor Universitário, 74605-050 Goiânia, GO, Brasil 3instituto de Saúde do Distrito Federal, Brasília, DF, Brasil ${ }^{4}$ Laboratório de Microbiologia, Instituto de Ciências Biológicas, Universidade de Brasília, Brasília, DF, Brasil ${ }^{5}$ Centro de Ciências Biológicas e da Saúde, Universidade Federal do Mato Grosso do Sul, Campo Grande, MS, Brasil ${ }^{6}$ Laboratório de Virologia Comparada, Instituto Oswaldo Cruz-Fiocruz, Rio de Janeiro, RJ, Brasil ’ Robert Koch-Institute, Berlin, Germany
\end{abstract}

Out of 1,588 faecal samples of children taken from three locations of the Central West Region of Brazil, 57 were positive for astroviruses (HAstVs) using reverse transcription-polymerase chain reaction (RT-PCR). They were genotyped by nested RT-PCR and/or genomic sequencing. HAstV-1 (42.8\%), HAstV-2 (23.2\%), HAstV-3 (3.6\%), HAstV-4 (14.3\%) and HAstVs -5, -6, -7 and -8 (1.8\% each) were detected. In Goiânia and Campo Grande, HAstV-1 was the most frequently detected genotype while in Brasilia (DF) it was HAstV-2. Shifts in the circulation of astrovirus genotypes were observed in DF and Campo Grande. All samples collected by rectal swabs were viral negative. The astrovirus genotypes were detected in all age groups and there was no correlation between genotype and age group.

Key words: astrovirus - genotyping - sequencing - nested-PCR - Brazil

Since human astroviruses (HAstVs) were described in 1975 (Appleton \& Higgins 1975), the medical importance of these viruses has been established and they have been shown to be the second most common cause of viral diarrhoea in young children (Herrmann et al. 1991, Cruz et al. 1992, Akihara et al. 2005). In Brazil, recent studies have described astrovirus detection rates as varying from 3.4-28.2\% (Gabbay et al. 2007, Resque et al. 2007, Santos et al. 2007, Victoria et al. 2007).

HAstVs have been classified into eight serotypes/genotypes (Matsui et al. 1998, Sakamoto et al. 2000). Previous studies have shown that HAstV-1 is the predominant type (Sakamoto et al. 2000, Mendez-Toss et al. 2004); however, simultaneous circulations of different astrovirus types in the same geographical area have been observed (Espul et al. 2004, Mendez-Toss et al. 2004).

In Brazil, there are few reports on the circulation of human astrovirus genotypes. They were done with surveillance data from cities such as Rio de Janeiro, São Paulo, Belém and Goiânia (Silva et al. 2001, Cardoso et al. 2002, Gabbay et al. 2007, Resque et al. 2007, Victoria et al. 2007). Information about human astrovirus

Financial support: CNPq (471968/03-0 and 520729/99-4). PAS was supported by DAAD (German Academic Exchange Service), CNPq, CAPES.

+ Corresponding author: dcardoso@iptsp.ufg.br

Received 28 April 2008

Accepted 31 March 2009 genotype circulation is important for the future development of prevention and control of astroviral infection. In this study, we describe the occurrence of astrovirus genotypes isolated from children with and without acute gastroenteritis carried out in three cities of the Central West Region of Brazil, including previously published data (Cardoso et al. 2002, Silva et al. 2006).

The subject of this study is the 57 astrovirus positive samples detected by reverse transcription-polymerase chain reaction (RT-PCR) (Cardoso et al. 2002) from 1,588 faecal samples (1,374 diarrheic and 214 non-diarrheic) collected from children under five years old that had been admitted to public hospitals from three locations of the Central West Region of Brazil because of diarrhoea [Brasília (DF) $(\mathrm{n}=501)$, Goiânia, Goiás $(\mathrm{n}=$ 743 ) and Campo Grande, Mato Grosso do Sul $(n=344)]$. Among the 57 positive samples, only one was from nondiarrheic samples. A sample was considered diarrheic if the faecal sample was collected from a child with acute diarrhoea, which was defined as three or more liquid or semi-liquid evacuations per day.

Faecal samples from DF were collected during two different periods of time, corresponding to 1994-1996 and 2000-2002. In the later period, the faecal samples were mostly (297/343) obtained by rectal swabs. In Goiânia, samples were collected between 1998-2002. In Campo Grande, samples were collected between 2000-2003. For 44 positive samples, genotyping of astroviruses was done by nested RT-PCR using primers that amplified the 3' end region of ORF 2 (Sakamoto et al. 2000). For determination of astrovirus genotypes by genomic sequencing, phylogenetic analysis over a $348 \mathrm{bp}$ region of the 5 , 
end ORF 2 was performed on 27 positive samples. The procedures are described in Silva et al. 2006. Fourteen samples were genotyped by both methodologies.

The nucleotide sequences for some of the samples detected in this study have been submitted to GenBank under the accession numbers DQ139825-DQ139832, DQ070852 and DQ028633.

This study was approved and conducted as recommended by the Ethical Committee on Human and Animal Research of the Federal University of Goiás (004/2000).

The genotyping of astroviruses performed by nested RT-PCR and/or genomic sequencing showed that all known viral genotypes (HAstV-1-HAstV-8) were detected in the Central West Region of Brazil. Samples genotyped by both methodologies $(n=14)$ are in agreement. Five samples (2 from DF, 2 from Goiânia and 1 from Campo Grande) that were submitted only to nested RT-PCR genotyping were untypeable, corresponding to $11.4 \%$ of the analysed samples. The genomic sequencing method successfully identified a viral genotype for all of the astrovirus-positive samples examined.

HAstVs $-1,-2,-3$ and -4 were detected in $42.8 \%$, $23.2 \%, 3.6 \%$ and $14.3 \%$ of diarrheic faecal samples, respectively, while the HAstVs $-5,-6,-7$ and -8 were each detected in $1.8 \%$ of diarrheic faecal samples. Considering the cities where the samples were obtained, it was observed that in DF the most frequent genotype was HAstV-2. In Goiânia and Campo Grande the HAstV-1 was predominant. The only non-diarrheic positive faecal sample was collected in DF in 2001 and it was characterised as HAstV-2.

In $\mathrm{DF}$, the HAstV-4 was the most frequent genotype in 1994 and 1995, while in 1996 the HAstV-2 was detected in $71.4 \%$ of the samples. This difference between predominant genotype and years of collection showed no statistical significance. Astroviruses were not detected in faecal samples from DF between 2000-2002. In Goiânia, HAstV-1 was the predominant genotype throughout all years of the study, except in 1999, when only two positive samples were detected, one was classified as HAstV-1 and the other as HAstV-8. In Campo Grande, HAstV-2 was predominant in 2000 and 2002, while HAstV-1 was the only one genotype detected in 2003 (Figure).

The different astrovirus genotypes were detected in children from all age groups up to five years old; however, the majority (41/57) were detected among young children - up to 24 months old. A correlation between genotype and age group was not observed $(p>0.1)$.

This study reports an overview of the distribution of astrovirus genotypes in three cities of the Central West Region of Brazil. It is the first time that all of the available astrovirus data obtained from this region between 1994-2003 are analysed together, allowing increased knowledge about the characteristics of the circulation of astrovirus genotypes.

Our data show that HAstV-1 is the most frequent genotype in the region. This finding corroborates the data of other authors from several regions of the world, including Brazil (Oh \& Schreier 2001, Cardoso et al. 2002, Gabbay et al. 2005, Resque et al. 2007). However,
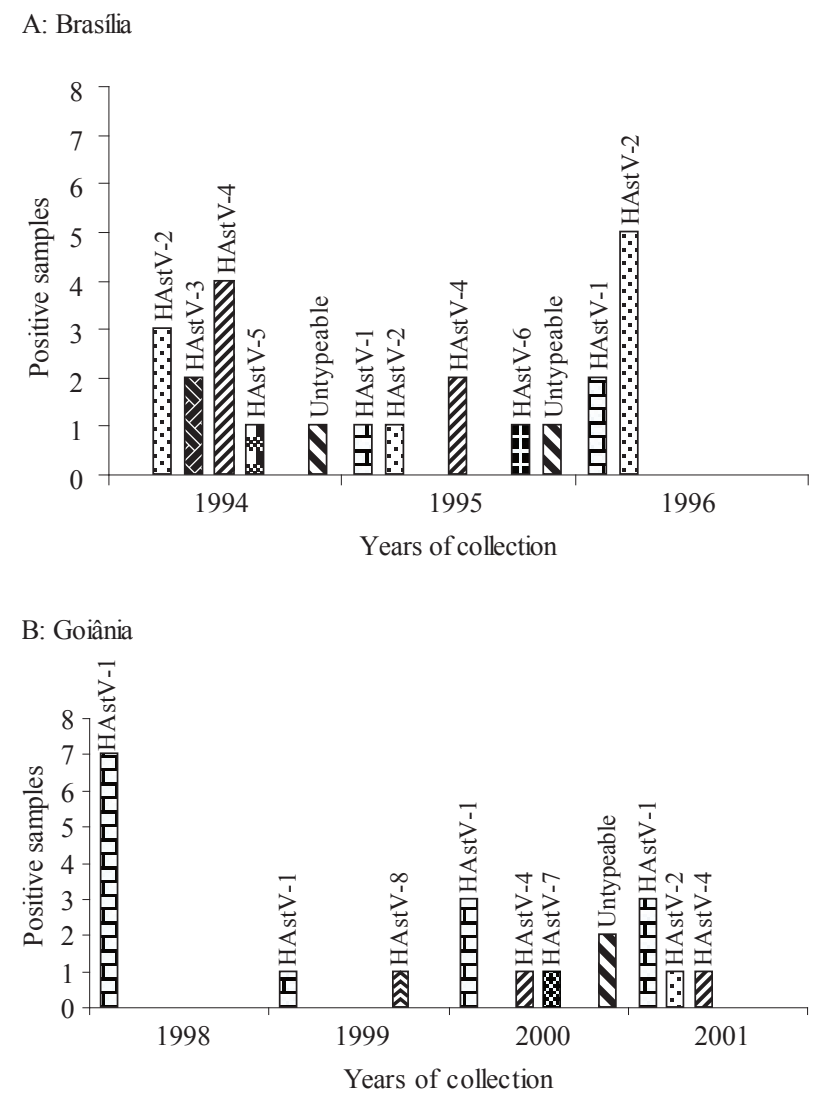

C: Campo Grande

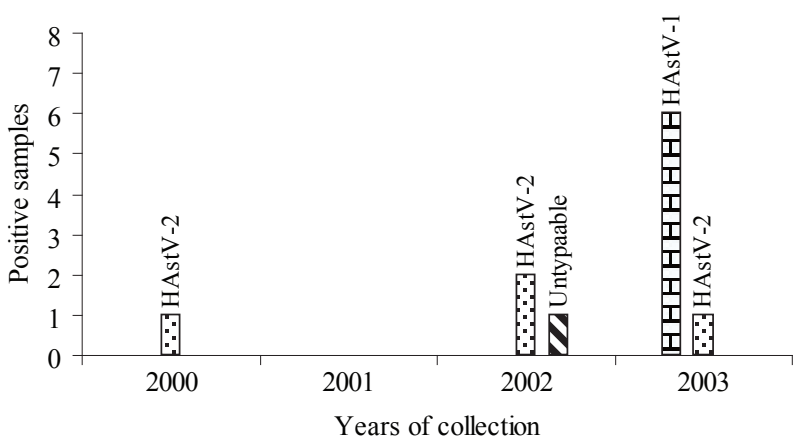

Distribution of astroviruses (HAstV) genotypes detected in Central West Region of Brazil, according to the location and year of collection.

other genotypes considered less common (HAstV-2HAstV-5) or rare (HAstV-6-HAstV-8) were also detected. The circulation of different genotypes in the same region is not uncommon and has also been reported in countries such as Germany, Spain, Argentina, Mexico and Egypt (Naficy et al. 2000, Oh \& Schreier 2001, Guix et al. 2002, Espul et al. 2004, Mendez-Toss et al. 2004). Recently, three studies carried out in large cities of Brazil showed the occurrence of at least four different genotypes in each region studied (Gabbay et al. 2007, Resque et al. 2007, Victoria et al. 2007). In the present study, the 
occurrences of at least five astrovirus genotypes were observed in DF and Goiânia, while only two genotypes were detected in Campo Grande. This data could be explained by the fact that these cities have higher immigration and tourism rates, facilitating the introduction of different astrovirus genotypes in the population. Besides the effect of migratory movements, we must also consider that the samples were collected in different intervals of time in each of the three locations and that this fact may have influenced the genotypic diversity of astroviruses among the cities.

Considering the positive rates of astrovirus detection, which were $4.8 \%, 2.8 \%$ and $3.2 \%$ in DF, Goiânia and Campo Grande, respectively (data not shown), no relation was found between astrovirus frequency and genotype diversity. This finding was also reported in Hungary, where a low prevalence rate $(1.6 \%)$ of astroviruses was found with a relatively high genotypic diversity (Jakab et al. 2004). In contrast, another study reported the circulation of only one genotype in a population with a higher prevalence $(9 \%)$ of this astrovirus (Liu et al. 2004).

In Goiânia and Campo Grande, the most frequent genotype was HAstV-1, while HAstV-2 was the most frequent in DF. This variation in genotype predominance from one location to another could suggest the occurrence of a regional predominance of astrovirus genotypes. This fact was also reported in other studies (Schnagl et al. 2002, Mendez-Toss et al. 2004).

The only one non-diarrheic positive faecal sample, from DF, was collected in a period when no positive diarrheic samples were detected, making comparisons between genotypes even more difficult. Comparisons with other periods of time demonstrated that the nondiarrheic positive faecal sample had the same genotype that was predominant in diarrheic faecal samples in DF - HAstV-2. In a study from Mexico, the same astrovirus genotypes were also detected from diarrheic and non-diarrheic children (Mendez-Toss et al. 2004). This finding suggests that there is no association between the infecting astrovirus genotypes and the presence or absence of diarrheic symptoms. Further studies with larger numbers of non-diarrheic and diarrheic faecal samples collected in the same period of time will certainly help to clarify this question.

Analysis of data obtained from positive samples from DF and Campo Grande in different years showed shifts in the circulation of astrovirus genotypes from HAstV-4-HAstV-2 and from HAstV-2-HAstV-1. This type of event has been observed in some studies with other viruses, including the gastrointestinal ones. Some authors have suggested that these shifts in viral genotypes could reflect a natural selection due to a lack of heterotypic immunity among the different viral antigenic types (McIver et al. 2000, Sakamoto et al. 2000, Guix et al. 2002, Sanchez-Fauquier et al. 2004, Maunula \& Von Bonsdorff 2005).

Clinical manifestations of astrovirus infection have been associated with vomiting, fever and abdominal pain (Walter \& Mitchell 2003). In this report, the analysis linking these symptoms and astrovirus genotypes was pos- sible only for samples from Goiânia (Santos et al. 2007) and correlation was not observed (data not shown).

Of 44 positive samples that were submitted to nested RT-PCR genotyping, five were untypeable because the DNA fragment sizes were inconclusive. This problem has been reported in another study, which suggests that the failure of nested RT-PCR typing is not related to nucleotide sequence variation, but probably to mismatches, inhibitors or low RNA yield (Walter et al. 2001).

Enteric viruses have usually been detected directly from faecal samples in natura, but other collecting methods, such as rectal swabs, have also been used (KhanMohammed et al. 2005). In this survey, we considered that the absence of positive astrovirus samples collected from DF between 2000-2002 could be attributed to the method of sample collection utilised. Most samples collected in this period were obtained from rectal swabs, which could inhibit viral detection due to the low RNA yield. This fact was also observed for caliciviruses (Borges et al. 2006). This finding shows the importance of the collecting method choice for the viral detection.

In this study there was no correlation between age group and astrovirus genotype, although previous data in Spain indicated a predominance of HAstV-1 and HAstV-3 in children under two years of age and HAStV-4 and HAStV-8 in children older than three (Guix et al. 2002).

In conclusion, the results of this research support the data that report the role of astroviruses as important agents of diarrhoea in children. However, they also provide the first data about the circulation of astroviral genotypes in three locations of the Central West Region of Brazil. This information is relevant to the implementation of future preventive strategies against HAstV infection.

\section{ACKNOWLEDGEMENTS}

To Dr. Wilia Marta Elsner Diederichsen de Brito, for the critical reading of the manuscript, and to colleagues from Laboratory of Virology in Goiânia-Brazil and from FG15, in Robert Koch Institute-Berlin, for the constant aid and support.

\section{REFERENCES}

Akihara S, Phan TG, Nguyen TA, Hansman G, Okitsu S, Ushijima H 2005. Existence of multiple outbreaks of viral gastroenteritis among infants in a day care center in Japan. Arch Virol 150: 2061-2075.

Appleton H, Higgins PG 1975. Viruses and gastroenteritis in infants. Lancet 1: 1297.

Borges AM, Teixeira JM, Costa PS, Giugliano LG, Fiaccadori FS, Franco Rde C, Brito WM, Leite JP, Cardoso DD 2006. Detection of calicivirus from fecal samples from children with acute gastroenteritis in the West Central Region of Brazil. Mem Inst Oswaldo Cruz 101: 721-724.

Cardoso DDP, Fiaccadori FS, Borges LD, Souza M, Bringel MRM, Gagliardi LJP 2002. Detection and genotyping of astroviruses from children with acute gastroenteritis from Goiânia, Goiás, Brazil. Med Sci Monit 8: CR624-628.

Cruz JR, Bartlett AV, Herrmann JE, Caceres P, Blacklow NR, Cano F 1992. Astrovirus-associated diarrhea among Guatemalan ambulatory rural children. J Clin Microbiol 30: 1140-1144.

Espul C, Martinez N, Noel JS, Cuello H, Abrile C, Grucci S, Glass R, Berke T, Matson DO 2004. Prevalence and characterization of astroviruses in Argentinean children with acute gastroenteritis. J Med Virol 72: 75-82. 
Gabbay YB, Linhares AC, Cavalcante-Pepino EL, Nakamura LS, Oliveira DS, da Silva LD, Mascarenhas JD, Oliveira CS, Monteiro TA, Leite JP 2007. Prevalence of human astrovirus genotypes associated with acute gastroenteritis among children in Belém, Brazil. J Med Virol 79: 530-538.

Gabbay YB, Luz CR, Costa IV, Cavalcante-Pepino EL, Sousa MS, Oliveira KK, Wanzeller AL, Mascarenhas JD, Leite JP, Linhares AC 2005. Prevalence and genetic diversity of astroviruses in children with and without diarrhea in São Luis, Maranhão, Brazil. Mem Inst Oswaldo Cruz 100: 709-714.

Guix S, Caballero S, Villena C, Bartolome R, Latorre C, Rabella N, Simo M, Bosch A, Pinto RM 2002. Molecular epidemiology of astrovirus infection in Barcelona, Spain. J Clin Microbiol 40: 133-139.

Herrmann JE, Taylor DN, Echeverria P, Blacklow NR 1991. Astroviruses as a cause of gastroenteritis in children. $N$ Engl J Med 324: $1757-1760$.

Jakab F, Meleg E, Banyai K, Melegh B, Timar L, Peterfai J, Szucs G 2004. One-year survey of astrovirus infection in children with gastroenteritis in a large hospital in Hungary: occurrence and genetic analysis of astroviruses. J Med Virol 74: 71-77.

Khan-Mohammed Z, Adesiyun AA, Swanston WH, Chadee DD 2005. Frequency and characteristics of selected enteropathogens in fecal and rectal specimens from childhood diarrhea in Trinidad, 1998-2000. Rev Panam Salud Publica 17: 170-177.

Liu CY, Shen KL, Wang SX, Liu YY, Zhaori GT 2004. Astrovirus infection in young children with diarrhea hospitalized at Beijing Children's Hospital. Chin Med J (Engl) 117: 353-356.

Matsui M, Ushijima H, Hachiya M, Kakizawa J, Wen L, Oseto M, Morooka K, Kurtz JB 1998. Determination of serotypes of astroviruses by reverse transcription-polymerase chain reaction and homologies of the types by the sequencing of Japanese isolates. Microbiol Immunol 42: 539-547.

Maunula L, Von Bonsdorff CH 2005. Norovirus genotypes causing gastroenteritis outbreaks in Finland 1998-2002. J Clin Virol 34: 186-194.

McIver CJ, Palombo EA, Doultree JC, Mustafa H, Marshall JA, Rawlinson WD 2000. Detection of astrovirus gastroenteritis in children. J Virol Methods 84: 99-105.

Mendez-Toss M, Griffin DD, Calva J, Contreras JF, Puerto FI, Mota F, Guiscafre H, Cedillo R, Munoz O, Herrera I, Lopez S, Arias CF 2004. Prevalence and genetic diversity of human astroviruses in Mexican children with symptomatic and asymptomatic infections. J Clin Microbiol 42: 151-157.
Naficy AB, Rao MR, Holmes JL, Abu-Elyazeed R, Savarino SJ, Wierzba TF, Frenck RW, Monroe SS, Glass RI, Clemens JD 2000. Astrovirus diarrhea in Egyptian children. J Infect Dis 182: 685-690.

Oh D, Schreier E 2001. Molecular characterization of human astroviruses in Germany. Arch Virol 146: 443-455.

Resque HR, Munford V, Castilho JG, Schmich H, Caruzo TA, Racz ML 2007. Molecular characterization of astrovirus in stool samples from children in São Paulo, Brazil. Mem Inst Oswaldo Cruz 102: 969-974.

Sakamoto T, Negishi H, Wang QH, Akihara S, Kim B, Nishimura S, Kaneshi K, Nakaya S, Ueda Y, Sugita K, Motohiro T, Nishimura T, Ushijima H 2000. Molecular epidemiology of astroviruses in Japan from 1995 to 1998 by reverse transcription-polymerase chain reaction with serotype-specific primers (1 to 8). $J \mathrm{Med} \mathrm{Vi-}$ rol 61: 326-331.

Sanchez-Fauquier A, Wilhelmi I, Colomina J, Cubero E, Roman E 2004. Diversity of group A human rotavirus types circulating over a 4-year period in Madrid, Spain. J Clin Microbiol 42: 1609-1613.

Santos RA, Borges AM, da Costa PS, Teixeira JM, Giugliano LG, Leite JP, Cardoso DD 2007. Astrovirus infection in children living in the Central West Region of Brazil. Mem Inst Oswaldo Cruz 102: 209-213.

Schnagl RD, Belfrage K, Farrington R, Hutchinson K, Lewis V, Erlich J, Morey F 2002. Incidence of human astrovirus in central Australia (1995 to 1998) and comparison of deduced serotypes detected from 1981 to 1998. J Clin Microbiol 40: 4114-4120.

Silva AM, Leite EG, Assis RM, Majerowicz S, Leite JP 2001. An outbreak of gastroenteritis associated with astrovirus serotype 1 in a day care center, in Rio de Janeiro, Brazil. Mem Inst Oswaldo Cruz 96: 1069-1073.

Silva PA, Cardoso DD, Schreier E 2006. Molecular characterization of human astroviruses isolated in Brazil, including the complete sequences of astrovirus genotypes 4 and 5. Arch Virol 151: 1405-1417.

Victoria M, Carvalho-Costa FA, Heinemann MB, Leite JP, Miagostovich MP 2007. Genotypes and molecular epidemiology of human astroviruses in hospitalized children with acute gastroenteritis in Rio de Janeiro, Brazil. J Med Virol 79: 939-944.

Walter JE, Mitchell DK 2003. Astrovirus infection in children. Curr Opin Infect Dis 16: 247-253.

Walter JE, Mitchell DK, Guerrero ML, Berke T, Matson DO, Monroe SS, Pickering LK, Ruiz-Palacios G 2001. Molecular epidemiology of human astrovirus diarrhea among children from a periurban community of Mexico City. J Infect Dis 183: 681-686. 